\title{
Conversion phenomenon during the induction period of general anesthesia -A case report-
}

\author{
Jin Seo Kim ${ }^{1}$, Eun Jung $\mathrm{Cho}^{1}$, Lee Jin Park ${ }^{2}$, and Jin Seok $\mathrm{O}^{1}$ \\ Departments of ${ }^{1}$ Anesthesiology and Pain Medicine, ${ }^{2}$ Neuropsychiatry, Incheon St. Mary's Hospital, College of Medicine, The \\ Catholic University of Korea, Incheon, Korea
}

Conversion disorder is characterized as psychological symptoms such as somatization and emotional distress, but there is no abnormal electrical signal in the brain. We report a patient who appeared conversion disorder during the induction period of general anesthesia. A 45-year-old woman was planned for arthroscopic knee meniscectomy. In the operating room, she appeared stable, but she said extremely nervous in this situation. Before propofol injection for induction of anesthesia, we injected $1 \%$ lidocaine $50 \mathrm{mg}$ iv for pain relief. Immediately after injection, she showed general seizure-like activity and then tonic-rigid muscle tone, dyspnea with periodic breathing without cyanosis, and clouding of consciousness. The operation was delayed, and she was examined by neurosurgeon and psychiatrist. She was diagnosed as suffering with conversion disorder and she was without brain abnormalities on the magnetic resonance imaging. Her condition improved after anti-depressant medication and supportive psychotherapy. She underwent uneventful knee surgery 40 days later. (Korean J Anesthesiol 2010; 59: 210-213)

Key Words: Anesthesia, Conversion, Distress, Psychotherapy.

Many patients experience preoperative anxiety prior to surgery, which can sometimes leads to psychological problems. Irritating matters are those concerned with death, nausea, vomiting, pain, urinary retention, postoperative confusion, nerve injury, and hypothermia [1]. The use of anxiolytic medication and counseling techniques have been shown to stabilize a patient's mood. Therefore, appropriate steps and careful assessment of mood status, intervention, and premedication can decrease the level of upsetting preoperative psychological conditions. Conversion disorder, once understood as hysteria, has a morbidity rate of $0.2 \%$ in the general population and a 5\% rate of occurrence in general hospitals. However, in some recent studies, life-long morbidity rates have been found to be as high as 33\% [2]. We report a patient who had previously undiagnosed anxiety, depressive disorder, and exhibited conversion phenomenon immediately after the injection of $1 \%$ lidocaine $50 \mathrm{mg}$ for anesthetic induction.

Received: August 24, 2009. Revised: 1st, September 8, 2009; 2nd, November 11, 2009. Accepted: November 17, 2009.

Corresponding author: Eun Jung Cho, M.D., Ph.D., Department of Anesthesiology and Pain Medicine, Incheon St. Mary's Hospital, College of Medicine, The Catholic University of Korea, Bupyeong-dong, Bupyeong-gu, Incheon 403-010, Korea. Tel: 82-32-510-5518, Fax: 82-32-505-8994, E-mail: gkjw2000@yahoo.co.kr

(c) This is an open-access article distributed under the terms of the Creative Commons Attribution Non-Commercial License (http:// creativecommons.org/licenses/by-nc/3.0/), which permits unrestricted non-commercial use, distribution, and reproduction in any medium, provided the original work is properly cited. 


\section{Case Report}

A 45-year-old woman was scheduled for arthroscopic left knee meniscectomy. Her preoperative medical history, hematological, and biochemical findings were within normal limits. Her weight, height, and body temperature were $80 \mathrm{~kg}$, $158 \mathrm{~cm}, 36.5^{\circ} \mathrm{C}$, respectively. Upon arrival to the operating room she appeared stable, but expressed fear and worry. Her blood pressure was 160/80 mmHg, heart rate 80 beats/min, and pulse oximetry saturation $100 \%$. Prior to propofol injection, $1 \%$ lidocaine $50 \mathrm{mg}$ was intravenously injected for pain control. Subsequently, she suddenly expressed hyperventilation, seizure like activity, then tonic-rigid muscle tone, dyspnea with periodic breathing (not involving cyanosis), profound sweating, and clouding of consciousness. She was ventilated with $100 \%$ oxygen $7 \mathrm{~L} / \mathrm{min}$ by mask. Her pupillary light reflex was normal, blood pressure was $170 / 110 \mathrm{mmHg}$, heart rate was $100-110$ beats/min, and electrocardiogram revealed a normal sinus rhythm. We prepared midazolam; however, this event stopped spontaneously after $2-3$ minutes. Her mental status returned to alert with the following vital signs, blood pressure 140/70 $\mathrm{mmHg}$, heart rate 70 beats $/ \mathrm{min}$, body temperature $36.4^{\circ} \mathrm{C}$, and a normal respiration rate. Sweating disappeared and she was able to obey commands. The operation was canceled, and she was sent to the recovery room. Except for a mild headache, her level of consciousness was alert, and she exhibited no neurological deficits on physical examination. In the recovery room, we carefully checked her medical history. It indicated that over the previous three years, several instances of sudden dyspnea, severe sweating attacks with mental change, had occurred during stressful events in her life.

She consulted with a neurosurgeon and psychiatrist. The magnetic resonance imaging (MRI) was normal however, upon psychiatric evaluation, she was diagnosed with anxiety, depressive disorder, and additionally, the cognitive function evaluation revealed lowered cognitive, attentive, and discriminative ability, and her IQ, as measured by the KoreanWechsler Adult Intelligence Scale, was 85 . Historically, she had experienced economic and familial problems. Together with clinical findings, we evaluated her as having conversion disorder.

After accurate reassurance coupled with reasonable rehabilitation to suit her symptoms, and with the suggestion of full recovery, further education, and drug treatment with milnacipran, buspirone, and lorazepam, her mood improved. Forty days later, she was subsequently readmitted for arthroscopic left knee meniscectomy. Upon arriving in the operating room, she indicated more comfort as compared to her previous experience. We injected midazolam $3.0 \mathrm{mg}$ immediately after arriving to the operating room, and she underwent the procedure on her left knee without incident under general anesthesia. Following up with appropriate oral medication through our psychiatric outpatient center resulted in symptomatic improvement.

\section{Discussion}

Patients attempt to escape from stressful circumstances in order to reduce anxiety, to ensure self protection, and to gain others' attention. Preoperative psychiatric evaluation could reduce anxiety problems prior to anesthesia, and thus, lead to improved outcomes [3].

Conversion disorder involves unbearable psychological symptoms to physical things as to resolve. Symptoms usually involve unconscious motor or sensory impairment and are not related to organic pathology $[2,4]$. Further, it is more frequent in women than in men. The onset of this disease is early adulthood, and tends to occur more frequently those living in rural areas, those associated with a lower socioeconomic class, and in less educated individuals [2]. In the past, our patient had experienced financial problems as well as major depressive disorder, which could be another causative factor for this disease.

The diagnosis of conversion disorder depends on psychological conditions that are not intentionally produced, and not on neurological or medical conditions [2]. Prior to diagnosis, physical evaluation, past medical history, and laboratory findings should be evaluated to exclude other diseases. Additionally, differential diagnosis excluding other organic etiological factors is important. Further, organic-related symptoms are physical stress-related, can occur at any time, and presenting systemic symptoms [4].

There are only a few reports of conversion related to anesthesia. In the operating room, conditions such as hypoglycemia, hypotension, hypothermia, hypercarbia, alkalosis, acidosis, electrolyte imbalance, and bleeding can delay awakening and thus, interfere with diagnosing conditions that are psychologically-induced [2,5]. Although conversion-evoked seizure may appear similar to episodes of true neurologicallybased seizure, those induced by conversion are not caused by an abnormal electrical discharges, transient ischemic attack, or hypoglycemia. In this case, the patient had no organic system damage, and further, her emotional stress led to chronic depression, which occurred only during times of emotional instability. The lack of skin cyanosis, retention of the pupillary light reflex, and negative sign on MRI indicated that her condition had not be of neurological origin [6]. We were unable to evaluate the patient's electroencephalography due to her refusal. After evaluation from a psychiatrist, it was determined that the patient's condition had originated from conversion 
disorder.

In the course of regional anesthesia, local anesthetics are used. At low concentrations in the blood, they serve to decrease cerebral blood flow, metabolism, and electrical activity, whereas at high concentrations, they act as proconvulsants by lowering seizure threshold. For example, lidocaine induces seizure by blocking brain inhibitory neurons in the amygdala and hippocampus [7]. Therefore, if a large amount of local agent is administered in regional anesthesia, patients can be at increased risk of CNS, cardiac toxicity problems. However, in this case, a total of only $50 \mathrm{mg}$ of $1 \%$ lidocaine was used, and therefore, the proconvulsant activity of lidocaine can be ruled out as the cause of the convulsion.

Factors associated with a good prognosis upon diagnosis of conversion disorder include being male, an acute onset, a short duration of symptoms, and a premorbid psychiatric diagnosis. Predictors of a poor prognosis include subclinical personality pathology, coexisting medical illness, and a poor perception of their own wellbeing [2]. In this case, the patient's disease interval period was three years, which is relatively long. Also, other mood disorders coexisted; however, her psychological conditions were well managed and symptoms were relieved through a outpatient clinic drug treatment program.

The management of conversion disorder involves a psychiatric examination and neurological assessment, followed by an explanation of the problem with successful communication and subsequent cognitive behavioral therapy. Additionally, preoperative education can lead to a better understanding of anesthesia and can thereby, decrease the related anxiety $[8,9]$. Further, preoperative medications such as alprazolam and midazolam can also reduce anxiety in preoperative patients [10]. In this case, we anesthetized the patient using midazolam $3.0 \mathrm{mg}$ immediately after her arrival to the operating room in order to decrease anxiety. A combination of psychotherapy, antidepressants, and the adherence to a multidisciplinary approach are beneficial in the resolution of this disease.

There has been nothing known regarding conversion disorder with respect to general and regional anesthesia. Also, there have been no case reports, such as the one presented here, of conversion phenomenon during anesthesia induction. Haden did report one case of an anxious 12-year-old girl whose tongue had been paralyzed after general anaesthesia for a dental procedure. Her symptom was resolved after counseling [11]. In another case, a spinal anesthetized patient was managed through propofol sedation in stressful arthroscopic surgical situation. After consulting with the pediatrician, it was concluded that preoperative stressful conditions had resulted in conversion disorder [12]. Further, Yokoyama et al. [13] reported a case of monoplegia conversion disorder after spinal anesthesia. In this patient, a psychic stressful family problem was the causative factor. Another case was reported involving a foot dorsiflexion defect after epidural anesthesia in an individual who had previously suffered from depressive mood disorder [14]. Clarke and Clarke [15] reported a 10-year-old girl with instant double vision problems after a dental block which was conclude to have originated from a fearful mind regarding the dental procedure and later disappeared after reassurance. It is vital to be prepared for any conversion circumstances using basic principles and anxiety-reducing sedation therapy regardless of whether general or regional anesthesia is employed. It is suggested that we sometimes miss psychological problems in patients and therefore, misdiagnose the problem in the operating room.

In conclusion, when patients present a sudden loss of sensory or abnormal motor function in the operating room, we should consider not only organic disease, but also conversion phenomenon. Careful evaluation of a patient's medical history, mental status, physical findings, and laboratory tests must occur. Overall, there is a need for releasing psychogenic stress during the preoperative period of anesthesia.

\section{References}

1. Macario A, Weinger M, Truong P, Lee M. Which clinical anesthesia outcomes are both common and important to avoid? The perspective of a panel of expert anesthesiologists. Anesth Analg 1999; 88: 1085-91.

2. Hollifield MA. Somatoform disorder. In: Comprehensive Textbook of Psychiatry. 7th ed. Edited by Sadock BJ, Sadock VA, Kaplan HI: Philadelphia, Lippincott Williams \& Wilkins. 2005, pp 1800-18.

3. Lee A, Gin T. Educating patients about anaesthesia: effect of various modes on patient's knowledge, anxiety and satisfaction. Curr Opin Anaesthesiol 2005; 18: 205-8.

4. Schecker N. Childhood conversion reactions in the emergency department: part II-general and specific features. Pediatr Emerg Care 1990; 6: 46-51.

5. Orr DL 2nd, Glassman AS. Conversion phenomenon following general anesthesia. J Oral Maxillofac Surg 1985; 43: 817-9.

6. Lindsay KW, Bone L. Neurology and neurosurgery illustrated. 3rd ed. London, Bath Press. 1997, pp 88-101.

7. Dorf E, Kuntz AF, Kelsey J, Holstege CP. Lidocaine-induced altered mental status and seizure after hematoma block. J Emerg Med 2006; 31: 251-3.

8. Moerman N, van Dam FS, Muller MJ, Oosting H. The amsterdam preoperative anxiety and information scale. Anesth Analg 1996; 82: 445-51.

9. Choi SJ, Kim GS, Choi YS, Kim JY, Kim CS, Gwak MS, et al. A survey of the nature of patients' preoperative anxiety and concerns regarding anesthesia. Korean J Anesthesiol 2002; 42: 11-6.

10. De Witte JL, Alegret C, Sessler DI, Cammu G. Preoperative alprazolam reduces anxiety in ambulatory surgery patients: a comparison with oral midazolam. Anesth Analg 2002; 95: 1601-6.

11. Haden RA. Conversion reaction following anaesthesia. Anaesthesia 
2004: 59: 728-9.

12. Sugimoto Y, Makino S, Doi Y, Nishimura M, Baba M, Mizukawa S, et al. A case of conversion disorder after spinal anesthesia. Masui 2009; 58: 209-11.

13. Yokoyama K, Okutsu Y, Fujita H. A case of monoplegia from conversion disorder after spinal anesthesia. Masui 2002; 51: 1363-7.
14. Diaz Allegue M, Gonzalez Bardanca S, Pato Lopez O, Abeledo Fernandez MA, Rama Maceiras P. Epidural anesthesia in labor and conversion disorder. Rev Esp Anestesiol Reanim 2009; 56: 312-4.

15. Clarke JR, Clarke DJ. Hysterical blindness during dental anaesthesia. Br Dent J 1987; 162: 267. 